\title{
The Application Study On The Customer Service Chatting Robot Based On Bot Framework
}

\author{
Chuanwei Qi \\ Puyang Vocational and Technical College \\ Puyang, China
}

Keywords: BOT FRAMEWORK; speech chatting robot; customer service.

\begin{abstract}
with the rapid development of speech recognition technology, the speech chatting robot has become as the breakthrough of the artificial intelligence. The voice chatting robot shall be such a typical application field in customer service, it can provide the customer with efficient and convenient service for 24 hours. In this study it develops an intelligent speech chatting robot for customer service by using the BOT FRAMEWORK released by Microsoft, the usage of such robot has greatly improved the the efficiency and satisfaction degree of customer service.
\end{abstract}

\section{CHATTING ROBOT REVIEW}

The chatting hereby mentioned refers to voice chatting. In comparison with text input, the voice chatting has so many advantages such as quick speed for inputting, convenient and easy to use, without spatial limit etc. Due to the lower recognition degree of computer on the voice previously, it may be quite hard for computer to understand the meaning of the natural language, it also obstructed the application of voice interaction. Along with the deepened development of machine learning technique, the computer's ability to process and recognize voice has been improved greatly, it's already possible for computer to correctly and precisely understand the voice instruction. In the artificial intelligent times, the voice intelligence has been become as the new breakthrough and also the tremendous development has also been gained in such field. Currently the intelligent voice chatting robot has been widely used in the fields of personal life assistant and commercial service such as knowledge Q \& A, weather inquiry, agenda reminding, traffic navigation, self-assistant catering and ticketing etc.

The intelligent voice robot has attracted the attention of foreign internet giants highly and they've also released their own relative products accordingly, such as Apple's Siri, Google's Google Now and Cortana, small ice released by Microsoft, Amazon's Echo intelligent speaker, Facebook's Messenger Platform, Tencent's QQ robot, Baidu's Xiao Du, Taobao's online customer service etc. The cognitive revolution raking the voice and language as the entry should must be the necessary path for the artificial intelligence. The application of intelligent voice chatting robot shall be more widely with brighter and brighter development prospect. One of the typical application field shall be the customer service. Because manual customer service can hardly realize the service within 24 hours at any time and anywhere; the online message also cannot response the customer with satisfied service due to its slow inputting speed and slow customer service response and processing. But the voice chatting robot for customer service could realize the service with high efficiency and convenience in 7 days for a week and 24 hours in a day, it should be very suitable for customer service field.

\section{BOT FRAMEWORK REVIEW}

BOT FRAMEWORK is such a framework released by Microsoft for constructing the intelligent chatting robot. It's a comprehensive product, to provide the simple and easy-to-use platform for developing the personalized intelligent chatting robot. The developer could use such framework to structure and deploy the robot with high quality, it enables the user having the voice chatting in high quality at any time and anywhere. Such framework includes Bot Builder SDK, Bot Connector, Developer Portal and Bot Directory. In addition, it also provides a simulator to test the developed voice chatting robot. BOT FRAMEWORK can understand the natural language and analyze the image etc. Initially it released 22 API that can be integrated in the application. The user can use these APIs to develop the personalized intelligent chatting robot..

It's worth noting that Microsoft's BOT was not such a robot based on hardware with the traditional meaning, but such a service framework similar to the online customer service. By using such framework it can realize the development in one time and the deployment of application in multiple places and times. This shall attribute to the convenience brought by Framework Channel. The BOT can be configured to following channels, see attached figure 1 .

\begin{tabular}{|c|}
\hline Channels \\
Email customer service \\
Short message service \\
Instant messaging \\
Web Chatting Room \\
\hline
\end{tabular}

Fig. 1. Channels 


\section{THE ESTABLISHMENT KNOWLEDGE LIBRARY}

Through calling the API interface of BOT FRAMEWORK, it can realize the common dialogue between the chatting robot and the user, because the robot of BOT FRAMEWORK has provided the function of intelligent chatting already, but robot of BOT FRAMEWORK shall be lack of professional knowledge of customer service, so we must realize the exclusive chatting robot for customer service through the way of establishing knowledge library of customer service. There are mainly 2 methods to establish the knowledge library as following.

\section{1 manual inputting}

The product information of model no, performance \& property, prices etc must be inputted or imported into the knowledge library manually; for those common questions most probably provided by the customer, it must determine the reasonable and scientific answer and explanation accordingly, and input it into the knowledge library accordingly. The information related to product and service in the customer service website must be inputted into the knowledge library. Of course the manual inputting may need plenty of manpower and material resources, and the inputted content may also be relatively limited, they can just match with the common questions. And people's ability on human knowledge reserve and ability is quite limited, to establish the knowledge library with mass information, it's obviously impractical to depend on manual inputting only, it must learn to access the knowledge from the internet.

\section{2 accessing knowledge from internet}

Today, we have successfully established the knowledge library in huge scale, such as plenty of websites and scientific literature in large scale like Baidu's encyclopedia, Baidu's library, Baidu knows, Zhihu, Quira etc. Because the contents in the Q \& A website were produced by the users with quite higher quality, and those knowledge were also closely related to human activities,otherwise the uses did not spend time and energies to ask such questions. Especially similar with the those websites like Baidu knows and Quora, while some users giving the answer, the other users can also vote on such answer accordingly, finally the best answer can be selected according to the number of voting. Those answers generated manually undoubtedly shall be better than that answers given by machine through automatic computing on the similarity. So that for the answers for common questions, we can fully utilize those knowledge and the existing answers for the common questions. In addition, there are also plenty of relative knowledge, discussion, Q \& A etc written by professionals in the professional platforms such as the professional forums, Weibo, Strategy etc, these contents can also be taken as the data of knowledge library. By utilizing these online data we can greatly improve the speed and quality of the knowledge library construction.
In case of the chatting robot cannot find out the suitable answer for the question given by the customer, the chatting robot can also search the answer from the internet, through analysis and sorting to provide the customer with the answer or relative links directly for customer's retrieval and screening. Most of the knowledge inside the chatting robot are managed in the way of index with searching engine, while taking the set of candidate answers, it may need to select the optimal answer or answers set to the user, this also makes the sorting becoming one of the important task. Although the ranking learning method in the search engine has had the reference meaning, the sorting characteristic in the search engine still cannot be copied mechanically as the sorting characteristics for the chatting robot system. We shall summarize all kinds of characteristics for sorting in the chatting robot, and revise the sorting model to train the machine learning system as our sorting model accordingly, to make the effort to answer the questions briefly, enable the chatting robot having more artificial intelligence accordingly.

\section{SYSTEM REALIZATION}

Bot Framework is such a simple framework based on ASP.NET MVC, it core is to develop such a simple web task, with the procedure of development as following:

\section{1 first to download a development template and simulator of Bot Framework.}

The development template can enable the developer to develop the BOT project in Visual Studio; the aim of simulator shall be to simulate and debug the developed voice chatting robot.

\section{2 installation of development template}

It's quite easy to install the template, just to copy a zipped pack into the template directory of vs, then to establish the BOT project inside the VS accordingly, as shown in figure 2.

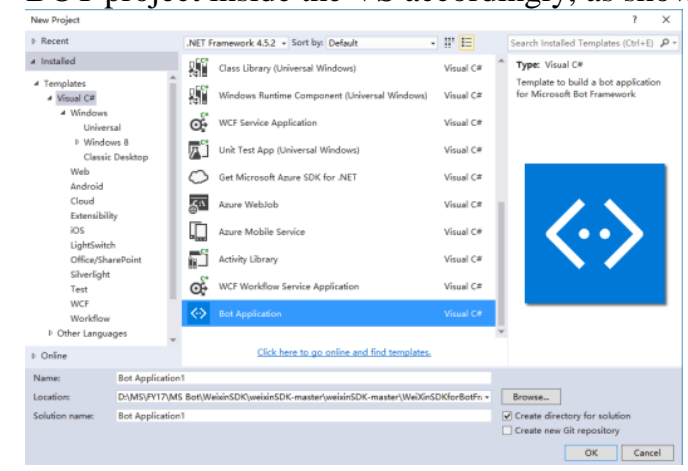

Fig. 2. Installing the development template.

\section{3 to register the developed BOT link on the portal of Microsoft's BOT FRAMEWORK}

The developed robot needs to be registered for use. The registration refers to describe the BOT to the framework, to access the application ID and password for the identity authentication of robot, it can register the developed robot 
through relative Microsoft website. While registering the service domain need to be deployed for the developed robot should be inputted, that is the address that MS BOT FRAMEWORK may transfer the message to, now the latest 3.0 version needs Microsoft App ID, the user may need to log on to MSA to create an APP ID accordingly. After filling up other information, to press the registration button then.

\section{4 to configure the web.config}

To add following configuration in the section of <appSettings> and </appSettings> in the web.config file of website.

$<$ appSettings>

$<$ !-- update these with your BotId, Microsoft App Id and your Microsoft App Password-->

$<$ add key="BotId" value="YourBotId" / >

$<$ add key="MicrosoftAppId" value="" />

$<$ add key="MicrosoftAppPassword" value="" />

$</$ appSettings>

Please note: the value of these two Keys are respectively MicrosoftAppId and MicrosoftAppPassword, they can both be found in BOT Portal.

\section{5 to configure the channel}

After the robot was registered, it may need to configure it to the channel used by the user, that is the configuration of channel. To configure the channel needs to be opened in the setting page of BOT: one channel shall be the chatting window of HTML; another channel shall be connected with the public account of WeChat. After the channel was configured, on one hand it can call such voice chatting robot in the chatting room of web page; on the other hand it can also use such voice chatting robot in the public account of WeChat.

Finally to call the simulator for debugging. Thus it can realize the purpose of using MS BOT FRAMEWORK meanwhile serving the public account of WeChat and Web chatting room of HTML accordingly, it then realized such an online intelligent customer-service chatting robot accordingly.

\section{SUMMARY AND EXPECTATION}

In this study it shows that by utilizing the BOT FRAMEWORK of Microsoft, it can rapidly and effectively develop an intelligent voice chatting robot used for customer service. Such robot can be used in multiple channels like the public account of WeChat, APP in mobile phone, web page chatting room, email customer service, instant messaging software etc. The actual application shows that the usage of such robot greatly improved the efficiency of customer service and customer's satisfaction, and also has had the good application prospect. In this study, the establishment of knowledge library mainly depended on manual collection and manipulation, by doing so the main problems shall be that it may need plenty of time and energies to collect, sort and input the knowledge library through the network, so that the cost of establishment and construction shall be quite higher, till now the size of constructed knowledge library was quite small, and the completed functions were also limited, so that the focus of next study shall be how to abstract the knowledge automatically from the network, enable the chatting robot performing self-learning accordingly.

\section{References}

[1] Z. Ji, Z. Lu, H. Li. An information Retrieval Approach to Short-text Conversation. arXiv: 1408.6988, 2014.

[2] Ritter A, Cherry C, Dolan W B. Data-Driven Response Generation in Social Media.[J]. Empirical Methods in Natural Language Processing, 2011:583-593.

[3] Ginzburg J, Fernandez R. Computational Models of Dialogue $[\mathrm{M}] / /$ The Handbook of Computational Linguistics and Natural Language Processing. Wiley Blackwell, 2010:429-481.

[4] Chai Qun, Zhang Shi-lu, Li Jia. Teaching laboratory research based on virtual reality simulation. [J]. Chinese education technology and equipment, 2009,4

[5] L. Shang, Z. Lu, and H. Li. Neural Responding Machine for Short-text Conversation. In Proceedings of ACL, 2015.

[6] L. Shang, Z. Lu, and H. Li. Neural Responding Machine for Short-text Conversation. In Proceedings of ACL, 2015.

[7] I.V. Serban., A. Sordoni, Y. Bengio et al. Building EndTo-End Dialogue Systems Using Generative Hierarchical Neural Network Models. arXiv:1507.04808v2 [cs.CL] 25 Nov 2015.

[8] Li Feng, Guo Geng-qi. Distance education platform based on J2EE and VRML research and implementation of key technologies. [J]. Modern computers (Professional Edition), 2009,1.

[9] Jurafsky, D., \& Martin, J. H. (2009). Speech and language processing an introduction to natural language processing, computational linguistics, and speech recognition. Prentice-Hall, Inc. Second Edition.

[10] Ananich, Anthony. "What is IaaS?". ananich.pro. Retrieved 2016-02-20.

[11] Rouse, Margaret. "What is public cloud?". Definition from Whatis.com. Retrieved 12 October 2014.

[12] Schmidt, Eric; Rosenberg, Jonathan (2014). How Google Works. Grand Central Publishing. p. 11. ISBN 978-1-4555-6059-2.

[13] Oestreich, Ken, (2010-11-15). "Converged Infrastructure". CTO Forum. Thectoforum.com. Retrieved 2011-12-02.

[author introduction] Chuanwei Qi (1970- ), male, born in Liangshan, Shandong, the mathematics and information engineering department of Puyang Vocational and Technical College, vice professor, with master degree, the research direction as the computer application technology and software engineering.

[Fund program] 2017 Henan science and technology research plan 